\title{
Multi-objective energy storage power dispatching using plug-in vehicles in a smart-microgrid
}

\author{
Vitor N. Coelho a, c, *, Igor M. Coelho ${ }^{\text {b, c }}$, Bruno N. Coelho ${ }^{c}$, Miri Weiss Cohen ${ }^{\text {d }}$, \\ Agnaldo J.R. Reis ${ }^{\text {f }}$, Sidelmo M. Silva ${ }^{\text {e, Marcone J.F. Souza }}{ }^{\mathrm{g}}$, Peter J. Fleming ${ }^{\mathrm{h}}$, \\ Frederico G. Guimarães ${ }^{\text {e }}$
}

a Graduate Program in Electrical Engineering, Universidade Federal de Minas Gerais, Belo Horizonte, Brazil

${ }^{\mathrm{b}}$ Department of Computer Science, State University of Rio de Janeiro, Rio de Janeiro, Brazil

${ }^{\mathrm{c}}$ Instituto de Pesquisa e Desenvolvimento de Tecnologias, Ouro Preto, Brazil

${ }^{\mathrm{d}}$ Department of Software Engineering, ORT Braude College of Engineering, Karmiel, Israel

e Department of Electrical Engineering, Universidade Federal de Minas Gerais, Belo Horizonte, Brazil

${ }^{\mathrm{f}}$ Department of Control and Automation Engineering, Universidade Federal de Ouro Preto, Ouro Preto, Brazil

${ }^{g}$ Department of Computer Science, Universidade Federal de Ouro Preto, Ouro Preto, Brazil

${ }^{\mathrm{h}}$ Department of Automatic Control and Systems Engineering, University of Sheffield, Sheffield, UK

\section{A R T I C L E I N F O}

\section{Article history:}

Received 1 April 2015

Received in revised form

27 November 2015

Accepted 30 November 2015

Available online 28 December 2015

\section{Keywords:}

Microgrids

Power dispatching

Energy storage management

Plug-in electric vehicle

Probabilistic forecast

Sharpe ratio

\begin{abstract}
A B S T R A C T
This paper describes a multi-objective power dispatching problem that uses Plug-in Electric Vehicle (PEV) as storage units. We formulate the energy storage planning as a Mixed-Integer Linear Programming (MILP) problem, respecting PEV requirements, minimizing three different objectives and analyzing three different criteria. Two novel cost-to-variability indicators, based on Sharpe Ratio, are introduced for analyzing the volatility of the energy storage schedules. By adding these additional criteria, energy storage planning is optimized seeking to minimize the following: total Microgrid (MG) costs; PEVs batteries usage; maximum peak load; difference between extreme scenarios and two Sharpe Ratio indices. Different scenarios are considered, which are generated with the use of probabilistic forecasting, since prediction involves inherent uncertainty. Energy storage planning scenarios are scheduled according to information provided by lower and upper bounds extracted from probabilistic forecasts. A MicroGrid (MG) scenario composed of two renewable energy resources, a wind energy turbine and photovoltaic cells, a residential MG user and different PEVs is analyzed. Candidate non-dominated solutions are searched from the pool of feasible solutions obtained during different Branch and Bound optimizations. Pareto fronts are discussed and analyzed for different energy storage scenarios. Perhaps the most important conclusion from this study is that schedules that minimize the total system cost may increase maximum peak load and its volatility over different possible scenarios, therefore may be less robust.
\end{abstract}

() 2015 Elsevier Ltd. All rights reserved.

\footnotetext{
This paper was intended as part of the special issue entitled "Optimization Methods in Renewable Energy Systems Design" Volume 87, Part 2, Pages 835-1030 (March 2016). However, the article was accepted after the deadline for acceptance for this issue and we were unable to delay compilation of the issue. We apologize for any inconvenience this may cause.

* Corresponding author. Department of Electrical Engineering, Federal University of Minas Gerais, Belo Horizonte, MG 31270-010, Brazil.

E-mail addresses: vncoelho@gmail.com, vncoelho@ufmg.br (V.N. Coelho), fredericoguimaraes@ufmg.br (F.G. Guimarães).
}

\section{Introduction}

The main goal of this paper is to address the power dispatching problem regarding to the minimization of six different objective functions: Microgrid (MG) total costs; usage of PEV batteries, maximum grid peak load, volatility behavior in extreme scenarios and two different criteria based on the Sharpe Ratio index. In order to evaluate suitable schedules to be applied in extreme scenarios, we make use of probabilistic forecasts to generate different scenarios. The multi-objective energy storage management problem considers PEVs as main storage units, located at SmartParks. Power 
dispatching schedule is planned to meet PEVs operational requirements, settled by its users, and trying to charge PEVs batteries when energy price is cheaper.

Energy storage has been studied over the last decades and remains a great challenge [1]. Especially in MG systems, its use has important benefits. The use of storage allows both sides, demand and production, to optimize the power exchanged with the main grid, in compliance with the electricity market and forecasts. Renewable energy generators associated with storage units are considered as active distributed generators, one of the fundamental elements of power management in MG systems. Current smartmicrogrid scenarios may include different renewable energy resources and different storage units. In this regard, storage is able to increase renewable energy self-consumption and independence from the grid. A wide range of applications exist for Energy Storage Systems (ESS). Tan, Li and Wang [2] refer the following: power quality enhancement, microgrid isolated operation, active distribution systems and PEVs' technologies. ESS ensembled with nondispatchable renewable energy generation units, such as wind and solar energy, can be mold into dispatchable units. Their use may improve dynamic stability, transient stability, voltage support and frequency regulation [3]. Furthermore, they can also be used for minimizing global cost and environment impact.

MG systems require smarter operations to well-coordinate these new emerging decentralized power energy sources. Optimization methods justify the cost of investing in a MG system by enabling economic and reliable utilization of resources [4]. Olivares et al. [5] observed that the microgrid optimal energy management problem falls, generally, into the category of mixed integer nonlinear programming problems. Because, in general, objective functions may include higher polynomial terms and operational constraints. Levron, Guerrero \& Beck [6] presented a methodology for solving the optimal power flow in MG. The model solves small systems containing up to two renewable generators and two storage devices. The proposed approach grows in complexity exponentially, since each storage device contributes extra dimensions to the solution space. The mathematical formulation proposed by Macedo, Franco, Rider \& Romero [7] extended the approach of Levron, Guerrero \& Beck [6]. Their formulation uses a convex equivalent model which obtains an approximate optimal solution for the same microgrid system. Mariani, Sareni, Roboam \& Turpin [8] researched the power dispatching problem seeking to minimize system global energy costs. A smart-microgrids DC system with flywheel energy storage was analyzed. By considering forecasts for a MG residence and solar PV production, an off-line power dispatching was performed in the search of storage planning schedules. Mohammadi, Soleymani \& Mozafari [9] considered uncertainties over the forecasting of consumption and renewable energy generation. A stochastic operation management of one day ahead was performed using a Heuristic Algorithm. At the initial state 2000 storage planning scenarios were generated, using a Probability Distribution Function (PDF) to represent the uncertainty of the forecasts. Those scenarios were generated and later reduced to 20 and sorted in ascending order of probability of occurrence. Recently, Kou, Gao \& Guan [10] integrated a battery ESS with a wind farm, using stochastic model predictive control scheme. Based on the forecasted wind power distributions and uncertainties, using a sparse warped Gaussian process, they sought for optimal operation regarding wind power dispatchability. The influence of wind power rapid ramp events was considered by Wang, Yu \& Yu [11], looking for an optimal dispatching strategy against wind power rapid ramp events during peak load periods. An energy storage system coupled with a PV plant was implemented for correcting the prediction errors by Delfanti, Falabretti \& Merlo in Ref. [12]. They tried to fulfill the lack between the injections of a PV power plant and the day-ahead market power schedule, minimizing energy imbalances.

Torreglosa et al. [13] analyzed a long-term energy dispatching, based on a model predictive strategy using on state control. Another long-term scheduling was evaluated by Tascikaraoglu et al. [14], considering a hybrid system with RER and energy storage, in the concept of virtual power plant. They analyzed the economic operation of the system in order to enable it to participate in the electricity market with high levels of reliable power production. Trovão \& Antunes [15] designed two meta-heuristic approaches for multi-ESS management in electric vehicles (EV). It has been noticed that hybridization of two or more energy storage elements into EV has been improving both the vehicle driving range and the lifecycle storage elements [16]. This kind of system allows batteries to perform power-sharing decisions in real time [17]. However, the latter did not consider the whole of RER along with the storage planning and scheduling.

Some approaches in the literature incorporated the reduction of Greenhouse gas (GHG) emissions as part of a Multi-Objective (MO) Optimization Problem [18-20]. Other applications spotlighted on finding the energy and power capacities of the storage system that minimizes the operating costs of the MG, as can be verified in Fossati, Galarza, Martín-Villate \& Fontán [21].

In this paper, a new multi-objective power dispatching problem is introduced, aiming to minimize global MG costs while minimizing saving batteries wear and tear, maximum peak load, volatility between extreme scenarios and schedule's total cost and maximum peak load volatility. Understanding the contributions of batteries as an objective function provides profits not only for the PEVs owners, but, also takes into account environment issues. Optimize its use not only reduces battery replacement costs for the PEVs owners but also is beneficial for the environment, since they are going to be used when needed. The proposed model also tries to obtain energy storage planning scenarios which minimize maximum power flow between the smart-microgrid and the main grid. The two latter objectives evaluate the schedule compared to its extreme scenarios and also to a wide range of possible scenarios. This is done by measuring the current expected cost compared to other possible costs using Sharpe Ratio [22]. Sharpe ratio is a useful index tool for analysis, used by investors facing alternative choices under uncertainties [23].

Different ESS have been adapted to be used over MG, some examples are: Battery Energy Storage System [6], Compressed Air Energy Storage systems [24], Flywheels [8], Thermal Energy Storage [25], Pumped-storage hydroelectricity [26], Superconducting Magnetic Energy Storage [27]. On the other hand, the use of energy storage in connection with SmartParks is becoming crucial demand as the number of PEVs, such as electric cars and plug-in hybrid, in the market is increasing [28]. Smart Grid applications, being developed, are still analyzing the benefits of this growth [29]. Power dispatching systems are incorporating vehicle-to-grid (V2G) power transactions over their schedule. Bidirectional power flow between PEVs and the grid will become essential [28,30]. As emphasized by Romo \& Micheloud [31], penetration of PEVs will increase significantly in the next 20 years. As a conclusion, smart parking lots with large fleets of electric cars can provide a flexible storage reserve for a MG system, reducing energy production needs.

Most of the work in the literature deal with the concept of parameters uncertainties of ESS management. In Papadopoulos et al. [32], results from a deterministic storage planning model showed that voltage violations would be quite high without the consideration of errors in the forecasts. From a probabilistic model with uncertainties, it was concluded that the integration of microgeneration in each MG household might reduce such violations. 
Previous works in ESS has focused on obtaining deterministic storage scenarios. This task was mainly done by introduction of uncertainty over forecasts and identifying the most likely scenarios $[25,8,9]$. Here, uncertainties are considered through the use of probabilistic forecasts, analyzing scenarios provided by their upper and lower bounds.

Probabilistic forecasts of MG components have been researched in the following areas: load [33], electricity prices [10,34], wind [35] and photovoltaic power [36,37]. Forecasting is a stochastic problem, probabilistic forecasts are able to provide additional quantitative information on the uncertainty associated with the MG components. Compared to currently wide-used deterministic forecasts, probabilistic forecasts are able to supplement point forecasts with probability information about their likely errors. Another advantage of using a probabilistic forecasting model is that they are able to quantify non-Gaussian uncertainties in wind and solar power forecasts. As analyzed by Zhang, Wang \& Wang [35], probabilistic forecasts are more appropriate inputs over decisionmaking in uncertain environments. It is expected that the use of probabilistic forecasts as inputs for energy storage management and power dispatching systems will become more widespread. The probabilistic forecasts provide reliable lower and upper bounds for each predicted time step, their use analyzing schedule in extreme scenarios is dealt with in this study.

In this work, a multi-objective ESS management problem with probabilistic forecasts is developed. Energy storage is studied on a smart-microgrid scenario composed of renewable energy generators, MG consumers and PEVs available at a SmartPark. The main goal is to optimize the total MG costs while minimizing the use of PEVs batteries, maximum peak load of the system and schedules' behavior in different scenarios. Operational requirements of the PEVs are considered: the specification of a desired percentage of energy in the PEVs during the storage schedule; the maximum Depth of Discharge (DoD) of batteries, in order to preserve the useful life of PEVs batteries. A smart storage scheduling model based on a mixed-integer mathematical formulation is designed. Non-dominated solutions are obtained from feasible solutions found over branches of the Branch and Bound (BB) optimization tree.

The major contributions of the current work are:

- Consideration of PEVs located at SmartParks as storage unit and respecting the operational constraints required by its users;
- To analyze the upper and lowers bounds provided by the probabilistic forecasts in order to test best-case and worst-case energy storage scenarios;

- A novel multi-objective power dispatching problem.

The remainder of this paper is organized as follows. Section 2 describes the microgrid scenario. Section 3 describes, in detail, the proposed energy storage management framework. Section 4 presents the computational experiments, and, finally, Section 5 details our final conclusions and future work.

\section{Microgrid scenario}

In the microgrid considered in this study, all components are connected through a DC bus without power flow constraints. The scenario is composed of:

- Consumption: A building with a maximum contractual power of $243 \mathrm{~kW}$.

- Production:

1. Wind Power Turbine (WPT) with a total capacity of $160 \mathrm{~kW}$;

2. Solar PV array with a total capacity of $80 \mathrm{~kW}$.

- SmartPark storage unit:

- PEV car composed with a typical Lithium-ion battery $60 \mathrm{~kW} /$ $60 \mathrm{kWh}$ storage.

- PEV car composed with three high speed flywheel $10 \mathrm{~kW} /$ $10 \mathrm{kWh}$ storage.

- PEV car composed with a CAES $60 \mathrm{~kW} / 60 \mathrm{kWh}$ storage.

The problem of energy management described here consists in planning, with a time step of $1 \mathrm{~h}$, energy storage for each hour of a desired planning horizon. Two different storage planning time horizons are handled in this current work, 24 and $168 \mathrm{~h}$ ahead.

Fig. $1 \mathrm{a}$ and $\mathrm{b}$ shows day and week month historical data of the analyzed periods. WPT data were adapted from EirGrid [38], Solar PV adapted from Hong, Wilson \& Xie [33] and residential house (adapted from Liu, Tang, Zhang \& Liu [39]). As can be verified in these figures, three different PEVs are showed. PEVs availability are stated between each pair of red and blue points (maybe a last red arrival point can be without pair, since vehicle will only departure later than the last time stamp). When vehicle arrives there is a red symbol marking its arrival state of charge (SOC). Analogously, in each departure, the blue point marks the desired battery SOC.

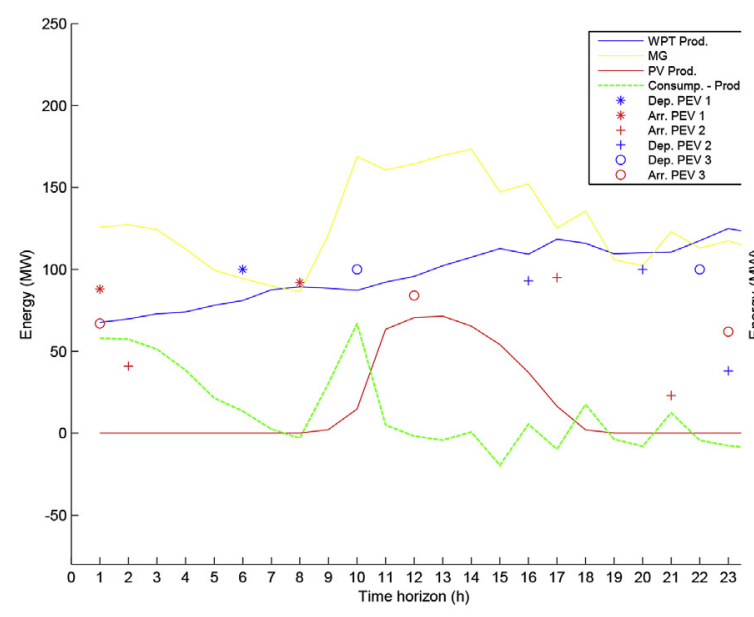

(a) One day.

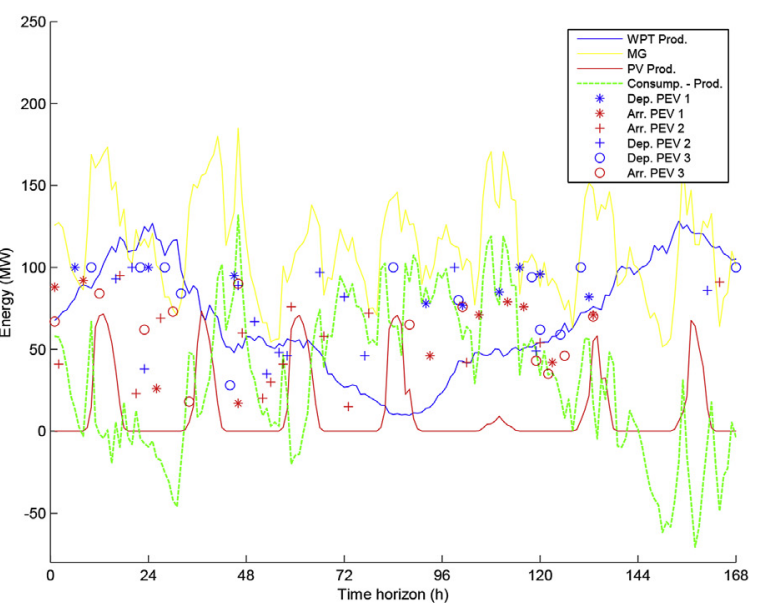

(b) One week.

Fig. 1. Historical microgrid data with hour sampling. 
During the arrival until the last time stamp before departure, PEV is available as an extra energy demand/source for the MG. Both words (demand/source) are used here since each PEV may represent an extra demand, taking into account that its owner might require charging during its stay at the SmartPark, what would represent an extra demand. On the other hand, if available to be used, as will be shown along this paper, it can represent a very useful and beneficial MG component.

The three PEVs depicted in Fig. 1a and b where generated according to the procedure described in Algorithm 1. arrival SOC. A maximum allowed percentage of charging per interval is set to be $35 \%$. Thus, any huge charging, higher than $35 \%$, is expected by the PEV owner. Parameters are formally presented in Section 3.2.

Typical microgrid prices, also obtained from Hong, Wilson \& Xie [33], are shown in Fig. 2. This figure shows the probabilistic forecast of the prices. In this case, the medium quartile $q_{50}$ is considered to be the real measured price. For simplicity, this data is repeated to the others days, when required by a longer energy storage planning.

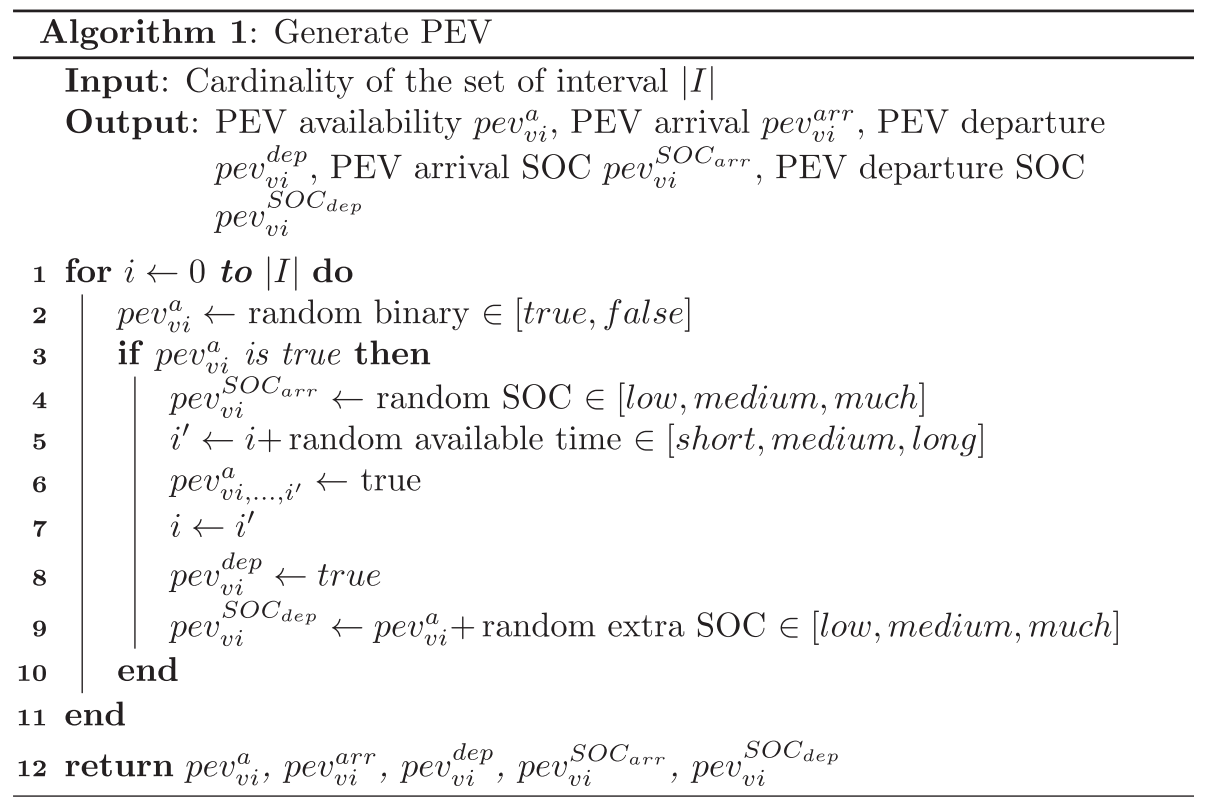

In Line 2 of Algorithm 1, PEV receives a random status of arriving or not. If it is arriving, a random initial SOC, from different ranges of possible initial SOCs, is assigned in line 4. After defining the availability time at the SmartPark, line 5, the departure flag is set in line 8 and a random departure SOC, higher than arrival, is defined in line 9. In this paper, each vehicle is considered to demand energy from the grid and, thus, its departure SOC is always greater than its

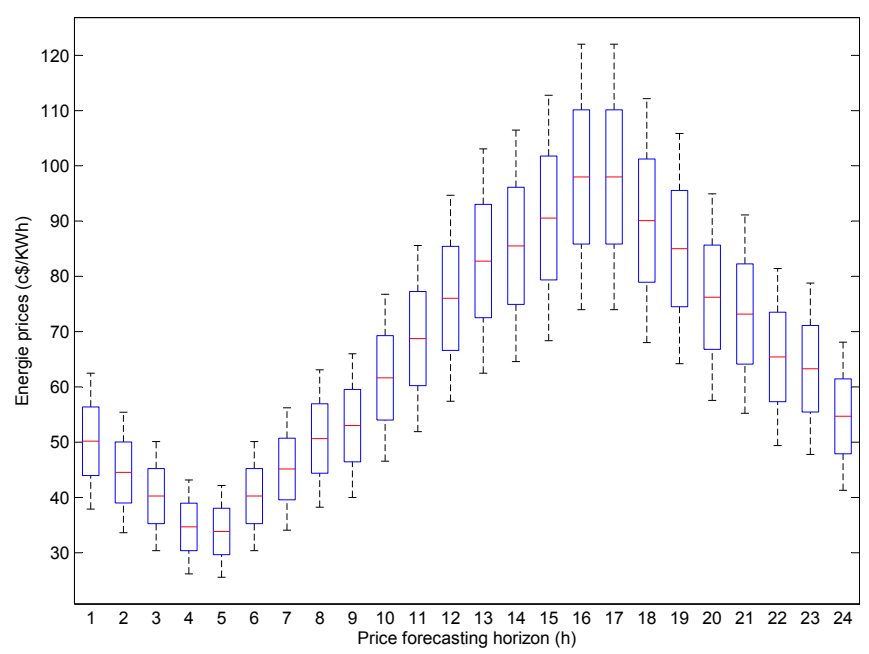

Fig. 2. Probabilistic price forecasts.

\section{Methodology}

This section describes the proposed framework developed and used to solve the multi-objective energy storage planning problem. First of all, Section 3.1 describes the model used to generate the probabilistic forecast for the MG components. Section 3.2 presents the mathematical formulation developed in this paper, as well as a description of the three main objective functions to be minimized. Section 3.3 introduces other criteria functions used to evaluate energy storage schedule behavior in extreme and different scenarios. Section 3.4 introduces the proposed Branch and Bound pool search algorithm.

\subsection{Probabilistic forecasting problems}

A set of $Q_{m g c}=\left[q_{1}^{m g c}, \ldots, q_{99}^{m g c}\right]$ probabilistic quartiles is considered for each microgrid component $m g c$ (energy consumption, wind and solar production, energy prices). Each quartile, $q_{i}^{m g c}=\left[f_{1}, \ldots, f_{t}, \ldots, f_{k}\right]$, is composed of a set of $f_{t}$ forecasts for the desired time horizon. The lowest and upper quartile $q_{0}$ and $q_{100}$ are not considered, since they are, technically, $-\infty$ and $\infty$.

The hybrid fuzzy heuristic algorithm of Coelho et al. [40] is adapted to perform the probabilistic forecast. Since the heuristic model is based on a fuzzy model calibrated using a bio-inspired metaheuristic algorithm, the proposal here is to change model parameters in order to generate different forecast values. Parameters changed here were the number of individuals of the population of Evolution Strategy [41] used to refine the fuzzy model which 


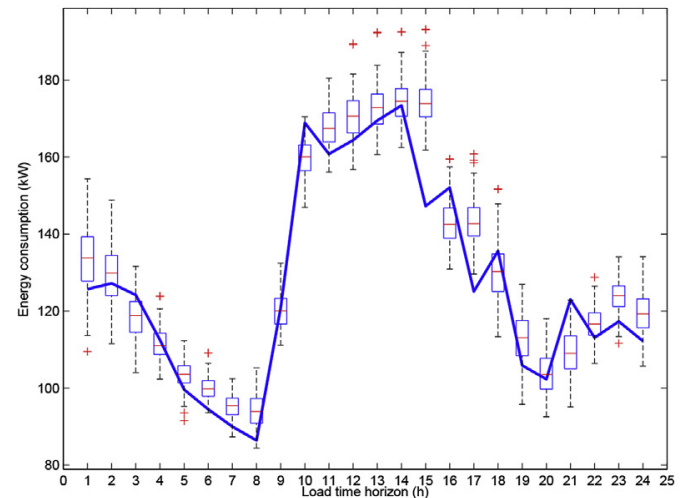

(a) Load consumption for one day ahead.

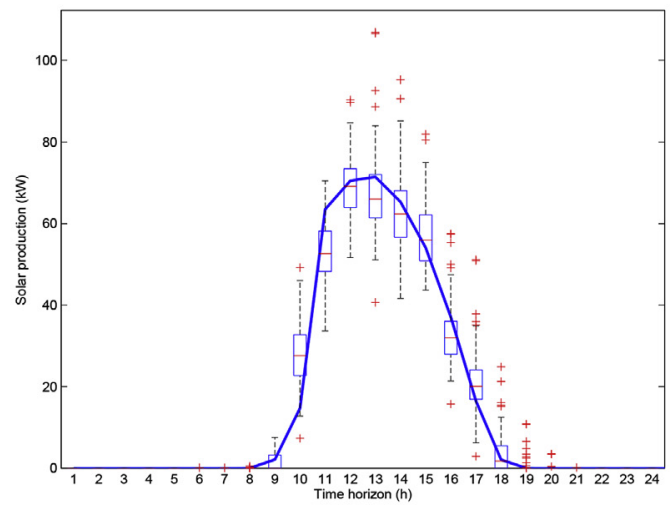

(c) Solar PV production

for one day ahead.

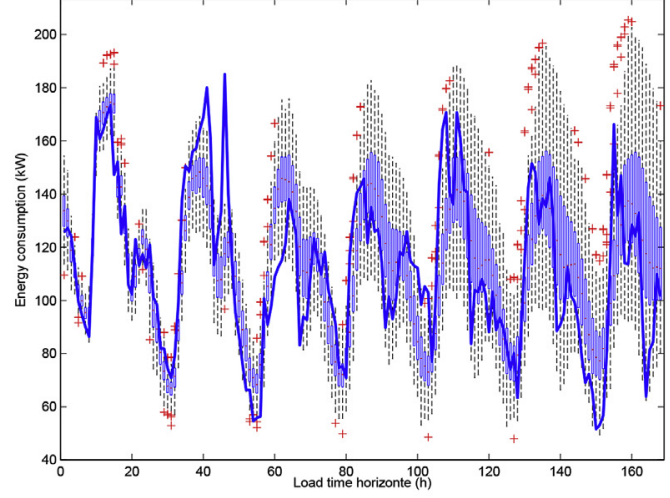

(b) Load consumption for one week ahead.

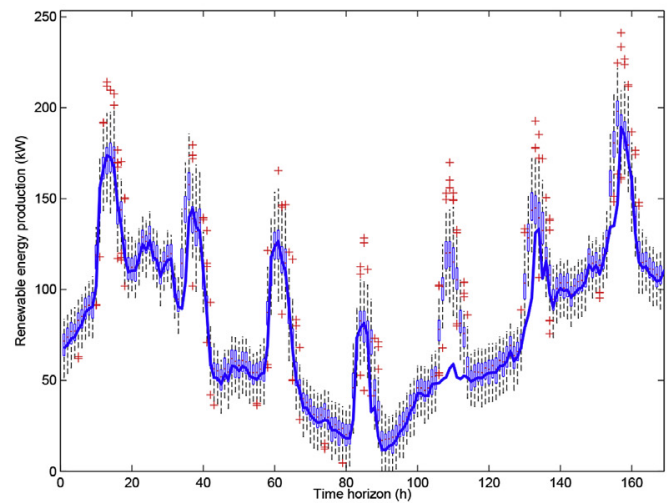

(d) Wind and solar generation for one week ahead.

Fig. 3. Probabilistic forecasts.

generates the forecasts. From the set of different forecast models, they were sorted from the lowest and highest values and quartiles were determined. If forecasts are far from the actual measured data, they are slightly adjusted in order to provide a reasonable probabilistic forecast scenario to be, didactically, used here.

Fig. 3a, b, 3c and 3d show the obtained probabilistic forecasts for the historical data introduced in Section 2. As can be verified, lower and upper quartiles ( $q_{1}$ and $q_{99}$, respectively) were able to afford acceptable limits for each MG component time series forecast (consumption (Fig. 3a and b), solar (Fig. 3d), renewable energy production, solar + wind, (Fig. 3d) and prices (Fig. 2)). From intervals the forecast time horizons 105 to 115 the model did not have a good performance in forecasting solar PV production, thus, a small gap can be verified. Nevertheless, since the extreme scenario analyses handled in this paper do not consider the relationship between the current measured values, the probabilistic forecast can still be considered precise.

\subsection{Multi-objective energy storage management problem}

A MILP model was developed in the interest of optimizing an global criterion based on the linear combination of three different objectives in energy storage planning. The following parameters were considered for the model:
I: Set of discrete intervals from 1 to furthest desired storage time horizon $k$;

$q_{i}^{d}$ : demand of all customers together at the interval $i \in I$;

$q_{i}^{r G}$ : indicates the energy production of all renewable energy resources at the interval $i \in I$;

$q_{i}^{\text {sell }}$ : energy selling price at the interval $i \in I$;

$q_{i}^{b u y}$ : energy buying price at the interval $i \in I$;

PEV: set of plug-in electric vehicles;

$p e v_{v}^{S O C_{\text {min }}}$ : indicates the minimum DoD of the vehicle $v$;

pev $v_{v}^{\text {Power }}$ : indicates PEV battery maximum capacity;

$p e v_{v i}^{a}$ : indicates if the vehicle $v$ is available at the SmartPark at the interval $i \in I$;

$p e v_{v i}^{a r r}$ : indicates if the vehicle $v$ is arriving at the SmartPark at the interval $i \in I$;

$p e v_{v i}^{S O C_{a r r}}$ : indicates the battery percentage of the vehicle $v$ at its arrival at the interval $i \in I$, obviously, if $p e v_{v i}^{a r r}=1$, otherwise it does not need to be attended;

$p e v_{v i}^{d e p}:$ indicates if the vehicle $v$ is departing from the SmartPark at the interval $i \in I$;

$p e v_{v i}^{S O C_{d e p}}$ : indicates the battery percentage demanded by the vehicle $v$ at its departure at the interval $i \in I$, if $p e v v i d$, otherwise it does not need to be attended;

$C$ : set of different battery cycles; pev $v_{v c}^{d R a t e}$ : battery discharging rate of the plug-in vehicle $v$ with power cycle $c$. 
pe $v_{v c}^{d P r i c e}$ : price for discharging the battery of the plug-in vehicle $v$ with rate $p e v_{v c}^{\text {dRate; }}$

pev $v_{v c}^{c R a t e}$ : indicates the charge rate of the vehicle $v$;

pe $v_{v c}^{c P r i c e}$ : price for charging the battery of the plug-in vehicle $v$ with rate of charge cycle pev $v_{v c}^{c R a t e}$.

The following decision variables were defined:

$e_{i}^{\text {sell }}$ : variable with real values indicating the amount of energy being sold at the interval $i \in I$;

$e_{i}^{\text {buy }}$ : variable with real values indicating the amount of energy being bought at the interval $i \in I$;

$e_{i}^{\text {sellActive }}$ : binary variable which indicates if any energy being sold at the interval $i \in I$;

$e_{i}^{\text {buyActive }}$ : binary variable which indicates if any energy being bought at the interval $i \in I$;

$y_{v i}^{b R}$ : variable with real values indicating the rate of battery of the vehicle $v$ at the interval $i \in I$;

$y_{v c i}^{c}$ : binary variable which indicates if the vehicle $v$ is charging with power cycle $c$ at the interval $i \in I$;

$y_{v c i}^{d}$ : binary variable which indicates if the vehicle $v$ is discharging with power cycle $c$ at the interval $i \in I$;

$t C D$ : real variable indicating the total charging and discharging expenses;

$f_{\text {objTotalcost }}$ : real variable indicating objective function that measures the MG total costs;

$f_{\text {objBatteriesuse }}$ real variable indicating objective function that measures batteries use;

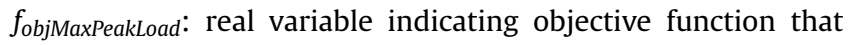
measures maximum peak load during the whole set of interval $i \in I$.

The mathematical model proposed in this paper can be seen from Eqs. (1)-(17). The global objective function to be minimized (Eq. (1)) is composed of the linear combination of three different objective functions, described in Eqs. (2)-(4). Total MG cost (Eq. (2)) is measured by the total amount of energy that is being bought or sold at each interval $i \in I$ plus the cost associated with each vehicle charge or discharge, these two latter are paid to the PEVs owners (its calculus is described in Eq. (8)). Batteries use (Eq. (3)) is figured by the sum of charges and discharges scheduled to perform during the whole energy storage planning. Eq. (4) attributes the maximum peak load of the MG system to the value of the third objective function.

Eqs. (5)-(7) force the system to only buy or sell energy at each interval. Eq. (9) forces the PEVs to only charge or discharge while Eqs. (10) and (11) make them charge or discharge only when PEVs are available at the SmartPark. Battery SOC limits, $p e v_{v}^{S O C_{\text {min }}} \leq y_{v i}^{b R} \leq 100$, are defined in Eqs. (12) and (13). Eq. (14) ensures that PEVs' batteries will attend a minimum SOC wished at its departure. PEV's battery rate is updated according to Eqs. (15) and (16). Eq. (15) attends the special case of the first interval while Eq. (16) takes the rate of the last battery, if the vehicle is not arriving, and add or subtract energy from charges or discharges. Finally, in Eq. (17), the amount of energy that is being sold or bought, at each interval $i \in I$, is determined.

minimize $\lambda_{1} f_{\text {objTotalCost }}+\lambda_{2} f_{\text {objBatteriesUse }}+\lambda_{3} f_{\text {objMaxPeakLoad }}$
S. T.:

$f_{\text {objTotalCost }}=\sum_{i \in I}\left(e_{i}^{\text {buy }} q_{i}^{\text {buy }}-e_{i}^{\text {sell }} q_{i}^{\text {sell }}\right)+t C D$

$f_{\text {objBatteriesUse }}=\sum_{i \in I} \sum_{v \in P E V} \sum_{c \in C}\left(y_{v c i}^{d}\right.$ pev $v_{v c}^{d R a t e}+y_{v c i}^{c}$ pe $\left.v_{v c}^{c R a t e}\right)$

$f_{\text {objMaxPeakLoad }} \geq e^{\text {buy }}+e^{\text {sell }} \quad \forall i \in I$

$e_{i}^{\text {sellActive } * M} \geq e^{\text {sell }} \quad \forall i \in I$

$e_{i}^{\text {buyActive }} * M \geq e^{\text {buy }} \quad \forall i \in I$

$e^{\text {sellActive }}+e^{\text {buyActive }} \leq 1 \quad \forall i \in I$

$t C D=\sum_{i \in I} \sum_{v \in P E V} \sum_{c \in C}\left(\left(y_{v c i}^{d}\right.\right.$ pe $v_{v c}^{d \text { Price }}+y_{v c i}^{c}$ pev $\left.v_{v c}^{c \text { Price }}\right)$ pev $\left.v_{v}^{\text {Power }}\right)$

$\sum_{c \in C}\left(y_{v c i}^{d}+y_{v i}^{c}\right) \leq 1 \quad \forall v \in P E V, i \in I$

$\sum_{c \in C} y_{v c i}^{d} \leq p e v_{v i}^{a} \quad \forall v \in P E V, i \in I$

$\sum_{c \in C} y_{v c i}^{c} \leq p e v_{v i}^{a} \quad \forall v \in P E V, i \in I$

$y_{v i}^{b R} \leq 100 \quad \forall v \in P E V, i \in I$

$y_{v i}^{b R} \geq p e v_{v}^{S O C_{\text {min }}} p e v_{v i}^{a} \quad \forall v \in P E V, i \in I$

$y_{v i}^{b R} \geq \operatorname{pev} v_{v i}^{S O C_{d e p}} p e v_{v i}^{d e p} \quad \forall v \in P E V, i \in I$

$\sum_{c \in C} y_{v 1}^{b R} \leq p e v_{v 1}^{S O C_{a r r}} p e v_{v 1}^{a r r} \quad \forall v \in P E V$

$$
\begin{gathered}
\sum_{c \in C} y_{v i}^{b R} \leq\left(1-p e v_{v i}^{a r r}\right) y_{v(i-1)}^{b R}+p e v_{v i}^{a r r} p e v_{v i}^{S O C_{a r r}} \\
+\sum_{c \in C}\left(y_{v c i}^{d} \text { pev } v_{v c}^{d R a t e}-y_{v c i}^{c} \text { pev } v_{v c}^{c R a t e}\right) \\
\forall v \in P E V, i \geq 2 \in I
\end{gathered}
$$

$$
\begin{gathered}
\sum_{v \in P E V} \sum_{c \in C}\left(\left(y_{v c i}^{d} \text { pe } v_{v c}^{d R a t e}-y_{v c i}^{c} \text { pev } v_{v c}^{c R a t e}\right) \text { pev } v_{v}^{\text {Power }}\right)+q_{i}^{r G}-q_{i}^{d}-\sum_{v \in P E V}\left(y_{v i}^{c} \text { pev } v_{v}^{c R a t e}\right) \\
=e_{i}^{\text {sell }}-e_{i}^{\text {buy }} \quad \forall i \in I
\end{gathered}
$$


Table 1

MG scenarios based on probabilistic quartiles.

\begin{tabular}{llll}
\hline \multicolumn{2}{l}{ Current MG energy scenario } & & \\
\hline Scenario & Consumption & Production & Price \\
\hline Worst case & $q_{99}$ & $q_{1}$ & $q_{99}$ \\
Best case & $q_{1}$ & $q_{99}$ & $q_{1}$ \\
Neutral & $q_{50}$ & $q_{50}$ & $q_{50}$ \\
\hline
\end{tabular}

\subsection{Extreme energy storage scenarios}

The energy storage schedule obtained by solving the mathematical model described in Section 3.2 is further evaluated regarding to six criteria. The first three criteria are the three objectives used in the optimization problem, while three additional criteria are introduced in this section.

The fourth criterion, so-called $f_{\text {objExtremescenario, evaluates the }}$ schedule compared to the opposite case of it. In other words, a comparison of the total cost of the worst and the best case is made and the discrepancy is returned. It seeks to find solutions which are flexible to be applied even in extreme scenarios, that is, this criterion measures the robustness of the schedule. Thus, batteries charge and discharge schedule are kept and analyzed through the most different expected scenario.

Table 1 indicates some possible MG scenarios based on energy consumption, renewable energy production and main grid energy price. As can be seen, the worst possible case, regarding to the total cost paid by the MG user, is the one when the consumption is the maximum possible $\left(q_{99}\right)$ with the highest expected prices $\left(q_{99}\right)$ and almost no renewable energy generation $\left(q_{1}\right)$.

Section 4 explores the results when a energy storage schedule is performed considering the worst case scenario and the best case scenario happens and vice versa.

The fifth and sixth criteria, namely $f_{\text {objSharpeRatioTotalcost, }}$

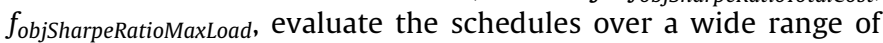
possible scenarios and use the Sharpe Ratio to verify the total cost and maximum load volatility. Eqs. (18) and (19) measure Sharpe Ratio, known in the literature as reward-to-variability index, but, here, adapted and used as a cost-to-variability indicator.

The schedule with the high expected cost and maximum peak loads is considered to be a constant risk-free return throughout the analyzed period. The optimum value for objective function $f_{\text {objBatteriesUse }}^{*}$ provides this information, since it represents the solution where energy storage is performed only seeking to attend
PEVs' constraints and save batteries use. This solution indicates an energy storage planning where all extra needed energy is bought from the main grid and the PEVs charge is scheduled to be done when the energy price is cheaper. In view that energy price can not guaranteed to be the cheapest, a small variability is also considered over $f_{\text {objBatteriesUse }}^{*}$ Thus, an adapted Sharpe Ratio

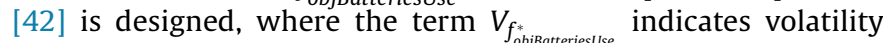
over the energy price (measured from probababilistic forecast variations from the time series depicted in Fig. 2). Finally, volatility $V\left(f_{\text {objTotalCost }}(s)\right)$ and $V\left(f_{\text {objMaxPeakLoad }}(s)\right)$ are obtained from the standard deviation of objective functions $f_{\text {objTotalcost }}(s)$ and $f_{\text {obj- }}$ MaxPeakLoad $(s)$, respectively, over a set of random scenarios. Random scenarios are generated from the combination of different quartiles of energy consumption, renewable energy production and energy prices. The behavior of the PEVs' scheduled charges and discharges of solution $s$ are analyzed for each of those scenarios.

$f_{\text {SRTotalCost }}(s)=\frac{f_{\text {objBatteriesUse }}^{*}-f_{\text {objTotalCost }}(s)}{V\left(f_{\text {objTotalCost }}(s)\right)-V_{f_{\text {objBatteriesUse }}^{*}}}$
$f_{\text {SRMaxPeakLoad }}(s)=\frac{f_{\text {objBatteriesUse }}^{*}-f_{\text {objMaxPeakLoad }}(s)}{V\left(f_{\text {objMaxPeakLoad }}(s)\right)-V_{f_{\text {objBatteriesUse }}^{*}}}$

\subsection{Branch and Bound pool search algorithm}

In order to obtain non-dominated solutions from the proposed MILP model, the use of solutions accessed in the BB [43] tree is considered. During the BB optimization over branches of its tree, different feasible solutions achieved during the searching procedure are saved in a pool of solutions. All these obtained solutions are considered to be inserted in the Pareto Front. In order to obtain solutions that optimize each objective function and the decision criteria ( $f_{\text {objTotalCost }}, f_{\text {objBatteriesUse, }} f_{\text {objMaxPeakLoad, }} f_{\text {objExtremescenar- }}$

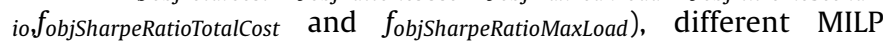
problems are generated by the linear combination of the weights $\lambda_{1}, \lambda_{2}$ and $\lambda_{3}$. Notice that since the problem is convex, any Paretooptimal solution regarding the objectives $f_{\text {objTotalcost }}, f_{\text {objBatteriesUse, }}$

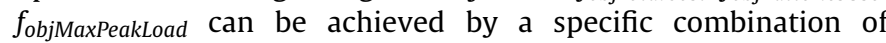
weights.

Algorithm 2 presents the procedure used to perform the linear combination and add solutions to the Pareto Front.

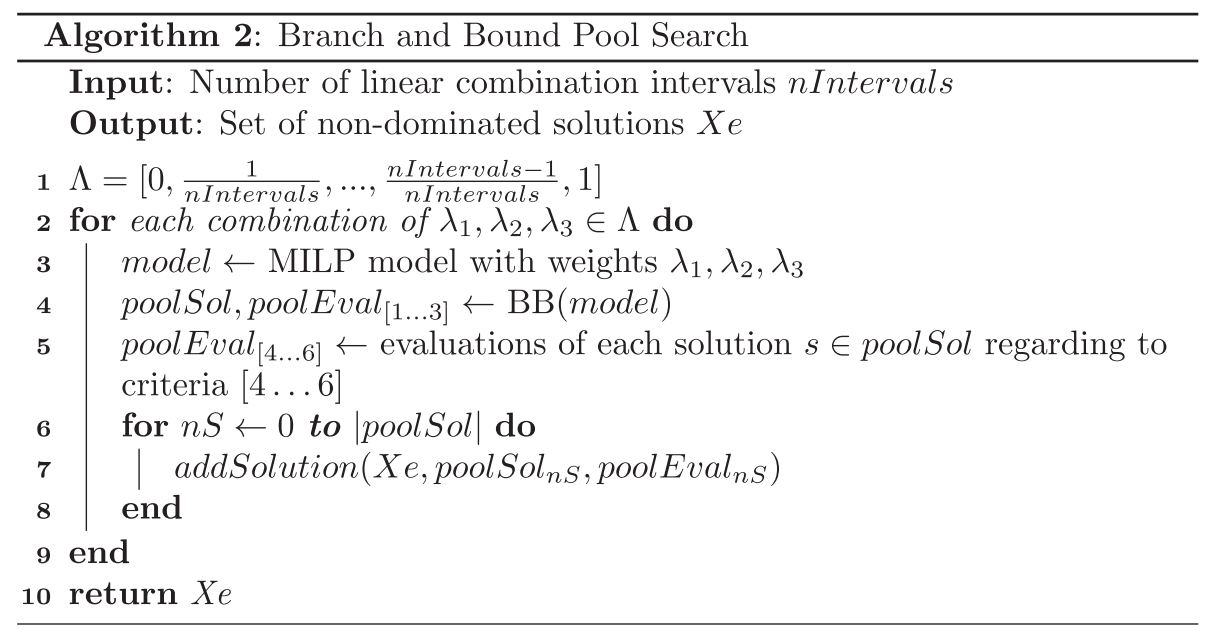



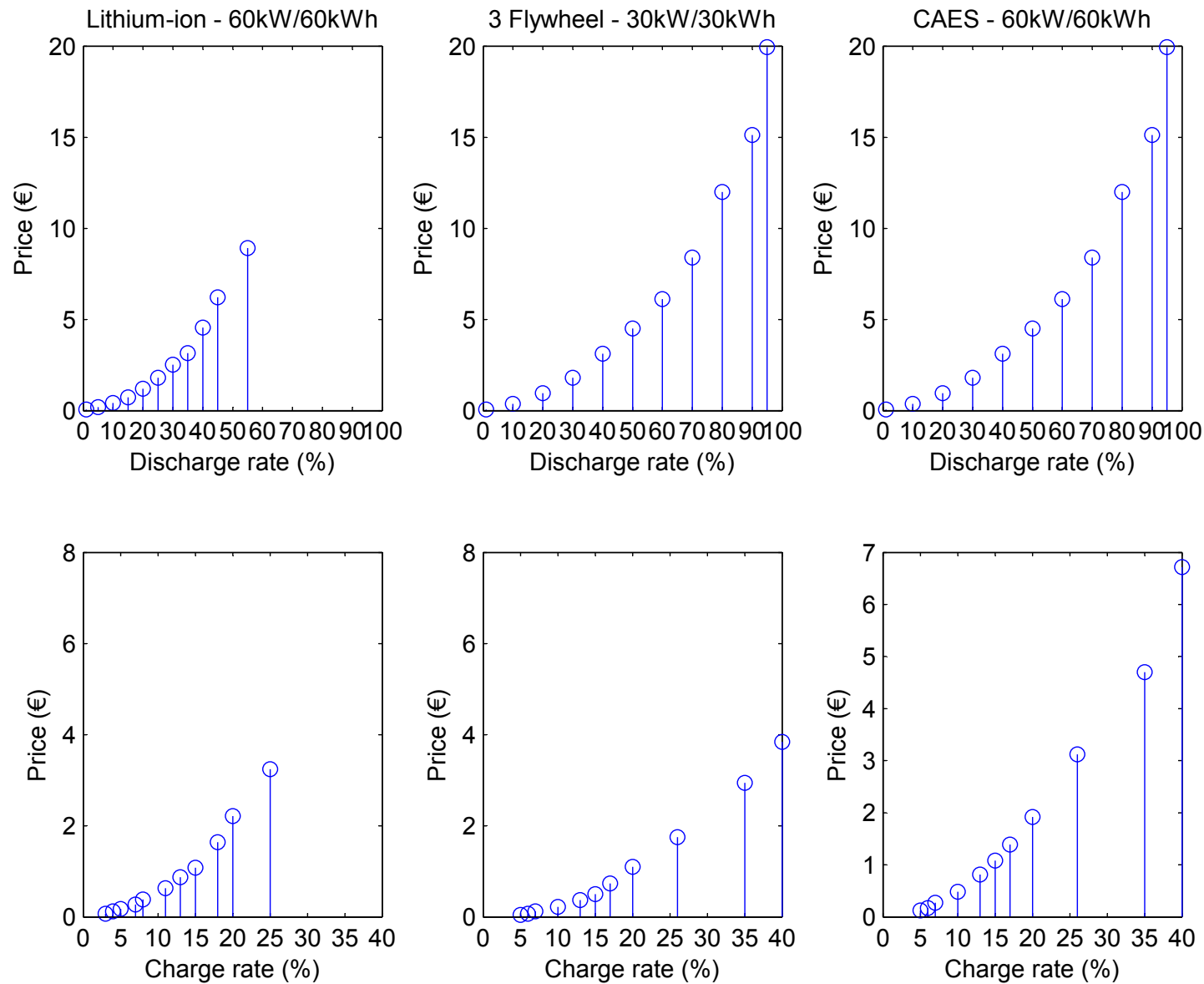

Fig. 4. Batteries rate of charge, discharge and prices.

Parameter nIntervals guides the precision of the linear combination between the weights $\lambda_{1}, \lambda_{2}$ and $\lambda_{3}$ and the number of solutions generated. A set of possible values for these weights, namely
$\Lambda$, is created in Line 1 of Algorithm 2. Basically, variable nIntervals regulates a discrete number of real values, from the interval $[0,1]$, that can be assigned to these weights.

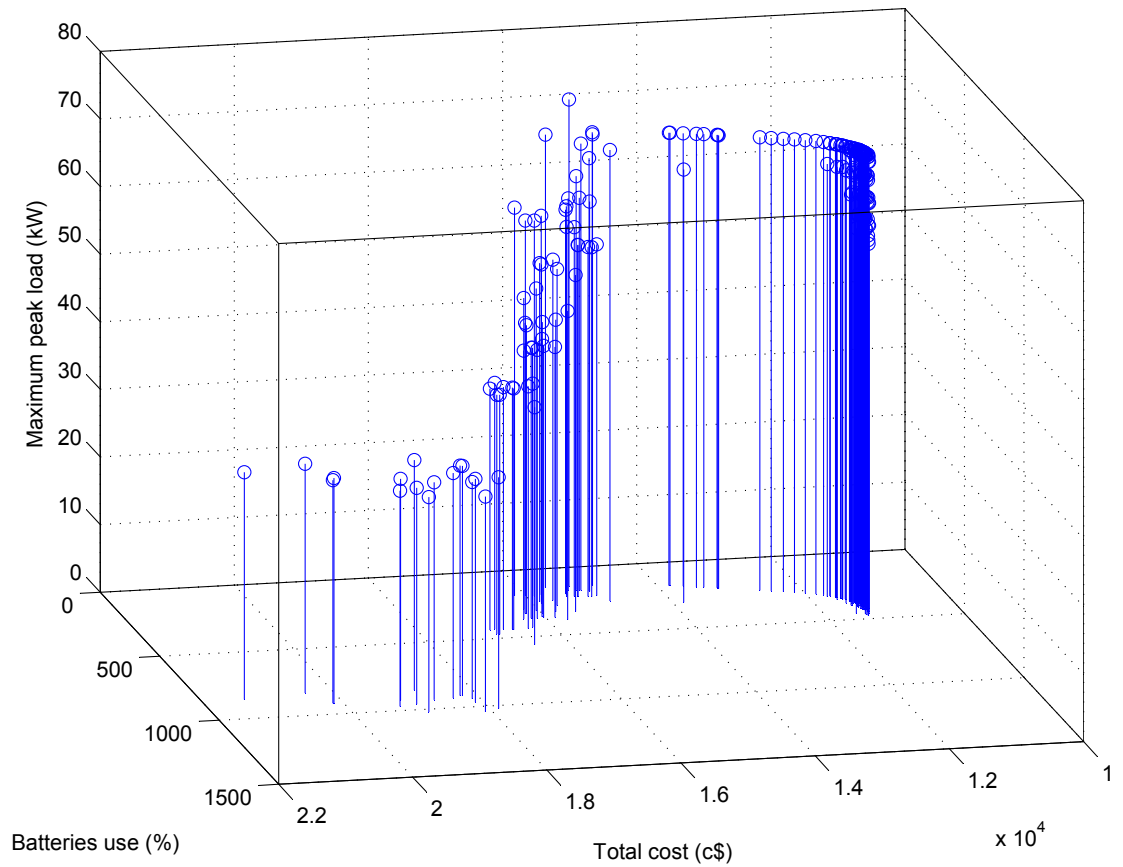

Fig. 5. Pareto front for one day ahead with deterministic energy storage schedule. 


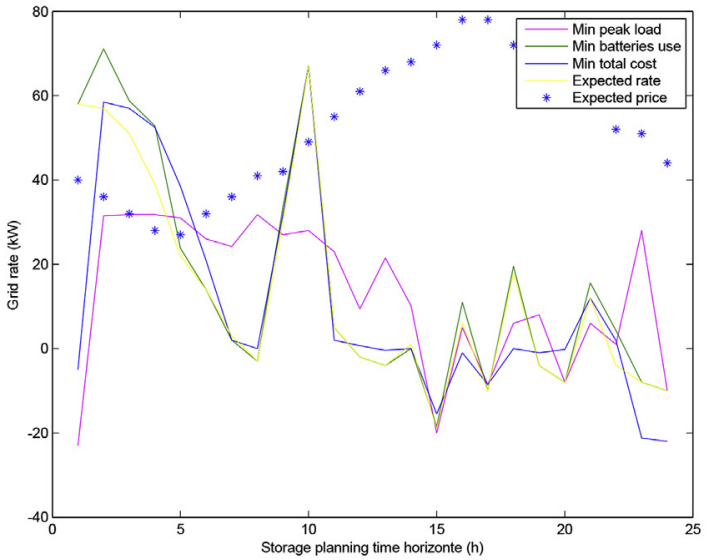

(a) One day ahead.

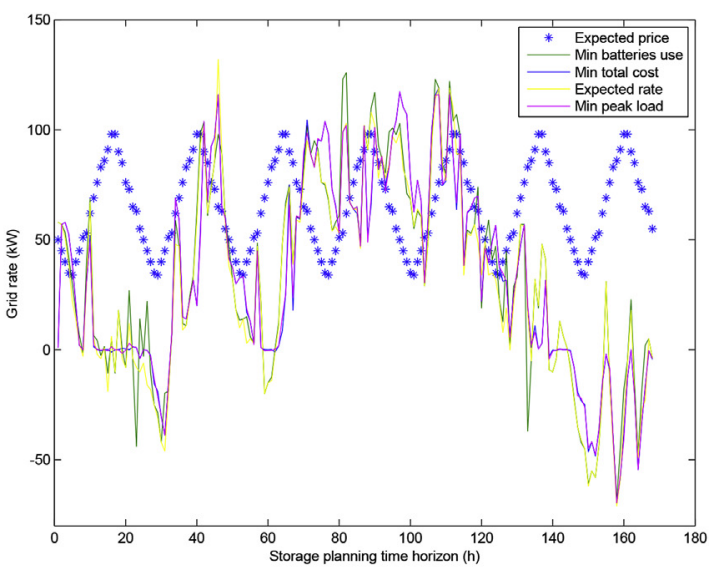

(b) One week ahead.

Fig. 6. Grid rate for deterministic power dispatching.

Line 3 of Algorithm 2 generates the math model described in Section 3.2 with weights $\lambda_{1}, \lambda_{2}$ and $\lambda_{3}$ for the objectives objTotalCost, objBatteriesUse, objMaxPeakLoad, respectively. The generated model is solved through a BB procedure (Line 4) and return obtained feasible solutions and its evaluations (regarding to the first three objective functions). Each solution from the pool is now evaluated according to the additional three criteria described in Section 3.3. Finally, the procedure addSolution (described in Algorithm 3), extracted from Lust \& Tehrem [44], is called in Line 7. This latter mechanism tries to add each obtained solution $s \in$ poolSol in the set of non-dominated solutions Xe. whole set of criteria, in which the results are analyzed using Aggregation Trees (AT) [45].

\subsection{Software and hardware configurations}

The BB pool search algorithm was implemented in $\mathrm{C}++$ in the framework OptFrame $2.0^{1}$ [46-48] running with CPLEX 12.5.1.

The tests were carried out on a DELL Inspiron Intel Core i7$3537 \mathrm{U}, 2.00 \times 4 \mathrm{GHZ}$ with $8 \mathrm{~GB}$ of RAM, with operating system Ubuntu 12.04.3 precise, and compiled by $\mathrm{g}++4.6 .3$, using the Eclipse Kepler Release.

each obtained solution $s \in$ poolSol in the set of non-dominated solutions $X e$.

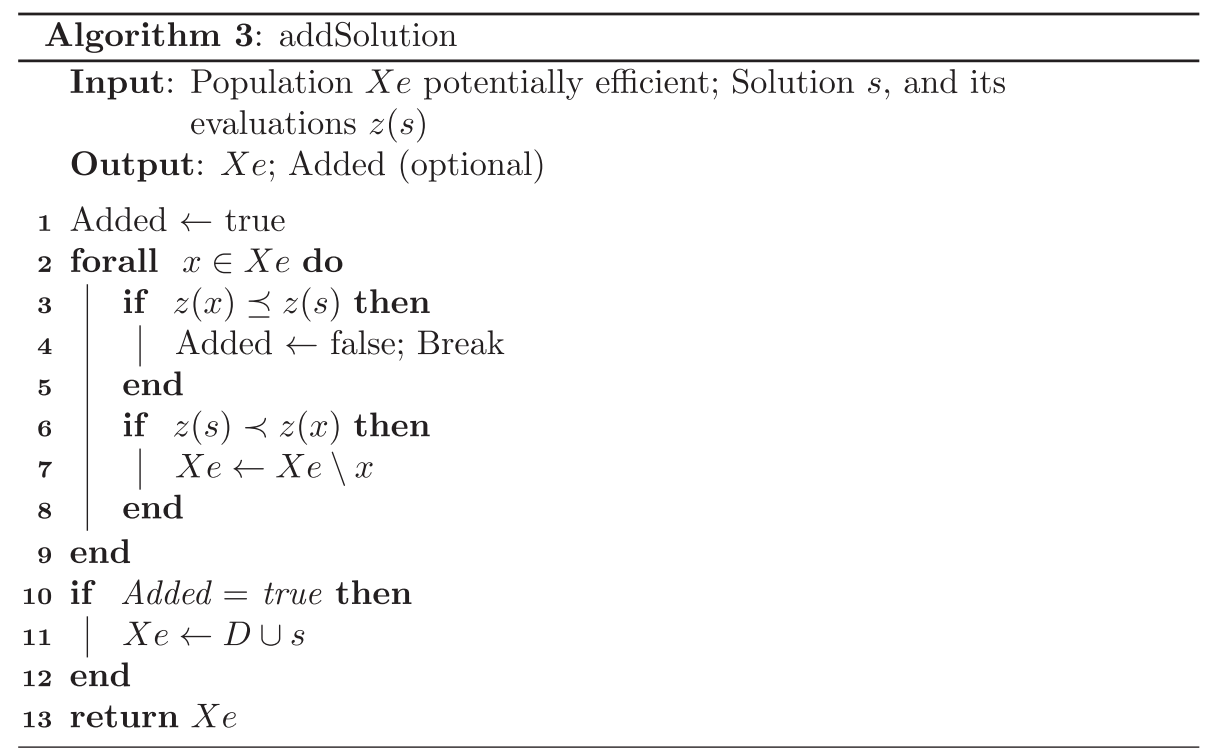

\section{Computational experiments}

This section is divided into three subsections. Section 4.1 presents the computational resources and some considerations about the model parameters. Section 4.2 describes the behavior of the first three objective function (criteria) over deterministic energy storage management using real measured historical data. Finally, Section 4.3 presents results of the proposed model regarding the

\subsection{Energy storage management over deterministic scenarios}

This first batch of experiments seeks to analyze the behavior of the proposed model over the deterministic scenario presented in Section 2. Two different storage planning time horizons were

\footnotetext{
${ }^{1}$ Available at http://sourceforge.net/projects/optframe/.
} 
evaluated, $k=24$ and $k=168$. Main grid prices of the first scenario were taken from the 11th quartile of the probabilistic forecast reported in Fig. 2. The expected buying prices for the forecast horizon of $k=168$ were taken from the medium quartile, $q_{50}$, and repeated for each day. Selling prices were set to be $70 \%$ of the buying price for the first energy storage planning and 30\% for the long-term. The number of discrete intervals nIntervals, which regulates the possible values for the objective functions weights (Section 3.4), was set to be 20 and 10 , respectively for $k=24$ and $k=168$. Thus, 9260 and 1330 MILP models were solved (excluding the case where $\lambda_{1}, \lambda_{2}, \lambda_{3}$ are equal to 0 ), respecting a maximum optimization time limit of $60 \mathrm{~s}$. For instance, the following set of possible values for the linear weightening were considered for the one-week ahead storage planning: $\Lambda_{k=168}=[0,0.1,0.2,0.3,0.4,0.5,0.6,0.7,0.8,0.9,1]$. As may be noticed, the number of possible values can be increased in large scale and real case applications by increasing the value of nIntervals.

Batteries characteristics are shown in Fig. 4. Flywheel and CAES batteries were set to be able to discharge deeper than the Lithiumion, $2 \%$ and $40 \%$ of maximum DoD, respectively. Possible rates of charge and discharge were generated according to 11 possibilities.

Fig. 5 presents the obtained set of non-dominated solution for the first forecast time horizon, composed of 205 solutions.

The expected grid rate for the best solution of each objective function can be seen in Fig. 6a and b. As can be verified, the optimization of each objective function resulted in different power dispatching strategies. The best total cost the one-day ahead schedule was $\$ 112.92$, with a total percentage of batteries use of $418 \%$ and maximum load of $67 \mathrm{~kW}$. By saving batteries use, a solution with a slightly greater maximum peak load of $72 \mathrm{~kW}$ was obtained with a total cost of $\$ 152.61$. The schedule which minimizes the maximum peak load schedule was able to minimize it in up to $31 \mathrm{~kW}$, expecting a total cost of $\$ 189,13$ and a total amount of batteries use equal to $1022 \%$. An analogous behavior was reported for the one week ahead storage planning.

\subsection{Energy storage management using probabilistic forecasts}

In this second batch of experiments, two different scenarios, extracted from Table 1, were considered. The first one involves power dispatching based on the worst case scenario and on evaluating objective function $f_{\text {objExtremescenario regarding to the best case. }}$ The second scenario was designed to optimize energy storage considering the best case scenario while its performance over the worst case scenario was also evaluated by $f_{\text {objExtremescenario. Sharpe }}$ ratio criteria $\left(f_{\text {objSharpeRatioTotalCost }}(s)\right.$ and $\left.f_{\text {objSharpeRatioMaxLoad }}(s)\right)$ were evaluated for 20 different random scenarios.

Fig. 7a, b, 8a, 8b, 9a and 9b present the obtained set of nondominated solutions, composed of more than 4000 solutions, represented by AT, polar and parallel coordinates Graphs as visualization tools for problems with many objectives (criteria).

As can be verified in the branches of the AT, considering the worst case scenario, criteria 3 and 6 and criteria 4 and 5 present low conflict, because these criteria were aggregated first in the AT. This result makes sense, it shows that minimizing the max peak load also tend to minimize the variability of the peak load. Moreover, in the worst case scenario the robustness of the total cost as measured by the criterion 4 is in harmony with the volatility measured by criterion 5 . On the other hand, objectives $f_{\text {objTotalcost }}(1)$ and $f_{\text {objBat- }}$ teriesUse $(s)$ (2) present the highest conflict, clearly capturing the trade-off existing in this power dispatch problem. For the best case scenario, criteria 1 and 2 still present the largest conflict since their groups are aggregated last in the AT. The relation of conflict and harmony between the other criteria can be similarly derived from the tree.

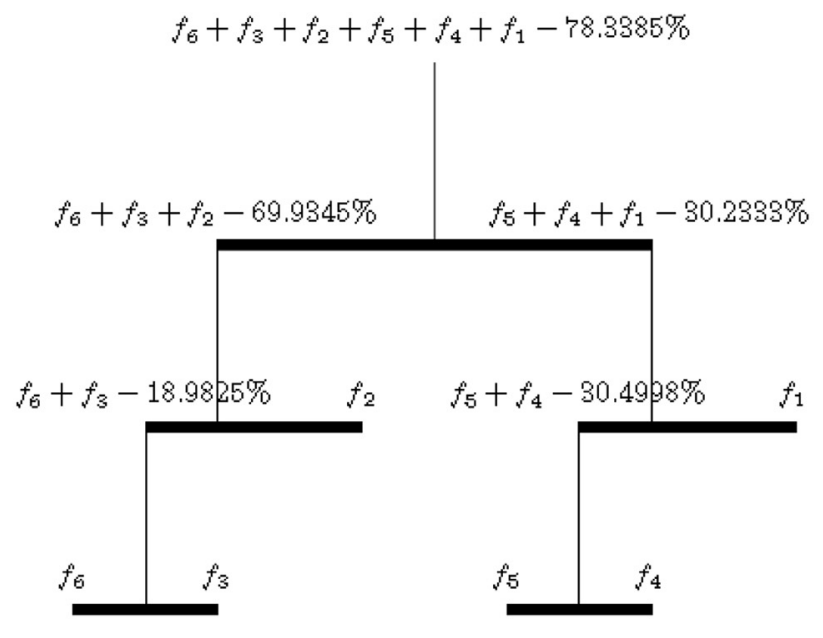

(a) Worst case storage planning.

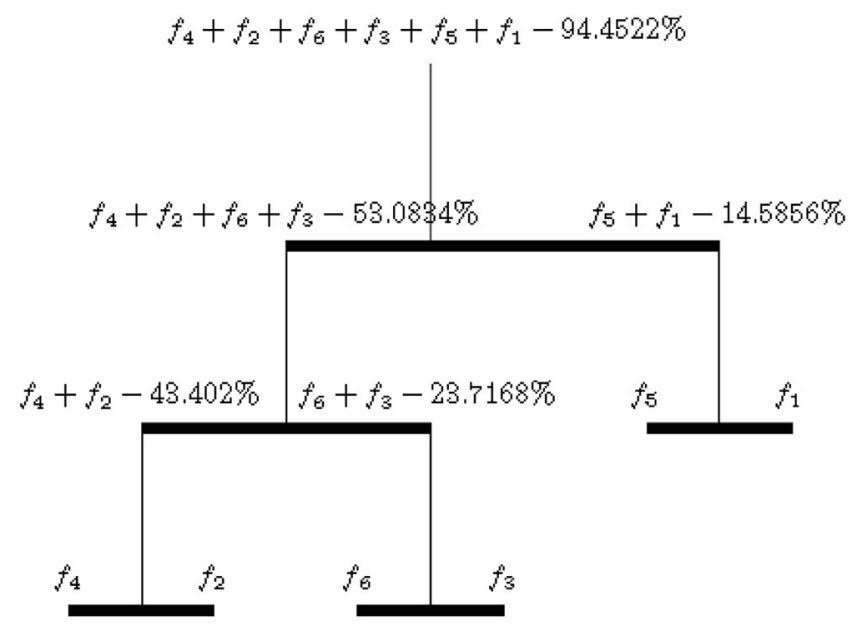

(b) Best case storage planning.

Fig. 7. Aggregation tree.

Since $f_{\text {objSharpeRatioMaxLoad }}(s)$ and $f_{\text {objMaxPeakLoad }}(s)$ are more harmonic criteria, it can also be concluded that PEVs batteries can be used for decreasing maximum peak load and its volatility over different possible scenarios. The use of PEVs batteries is also beneficial for reducing the difference between the expected total cost of the power dispatching and the one that might happen in extreme scenarios.

\section{Conclusions and extensions}

\subsection{Summary and final considerations}

In this paper, a novel multi-objective energy storage power dispatching was analyzed and discussed. Optimization of different MG characteristics was proposed, such as: MG total costs, use of PEVs batteries, maximum MG system peak load, behavior in extreme and sets of different scenarios. Probabilistic forecasts were used in order to evaluate energy storage schedule in extreme 
Polar coordinates trade-off graph

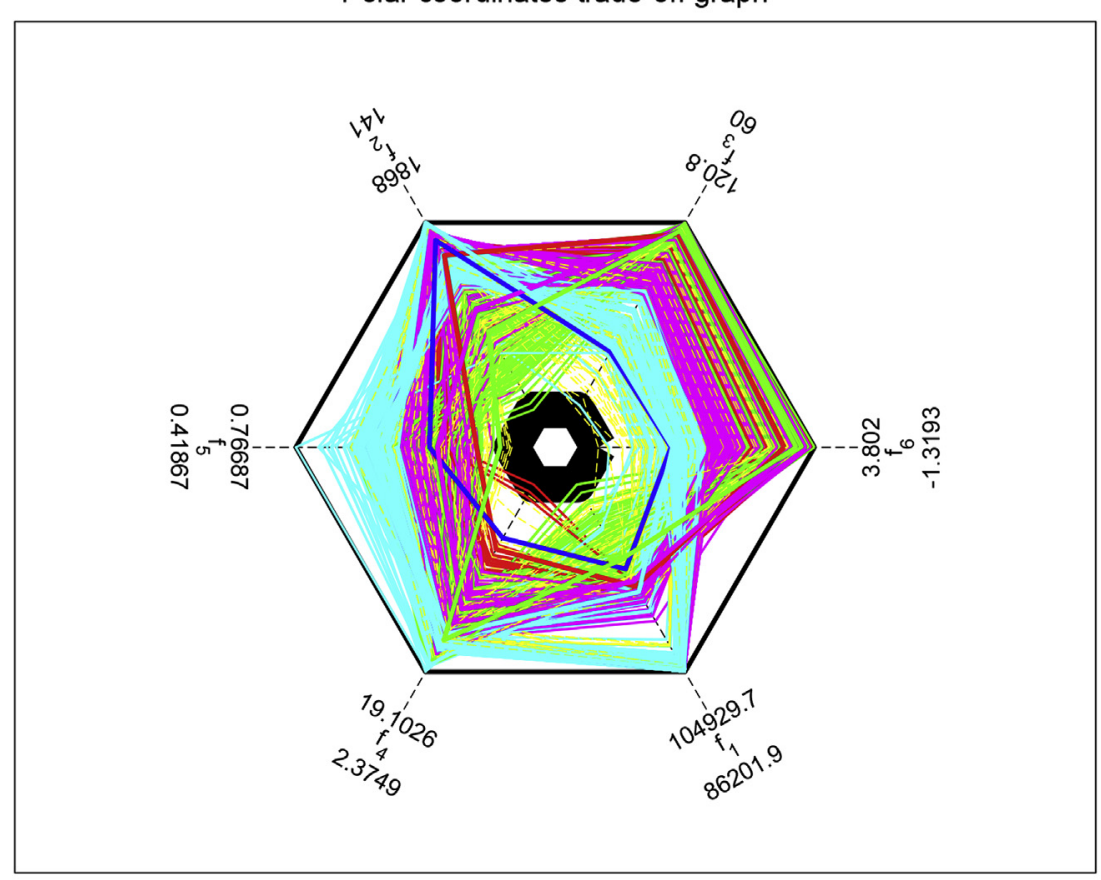

(a) Worst case.

Polar coordinates trade-off graph

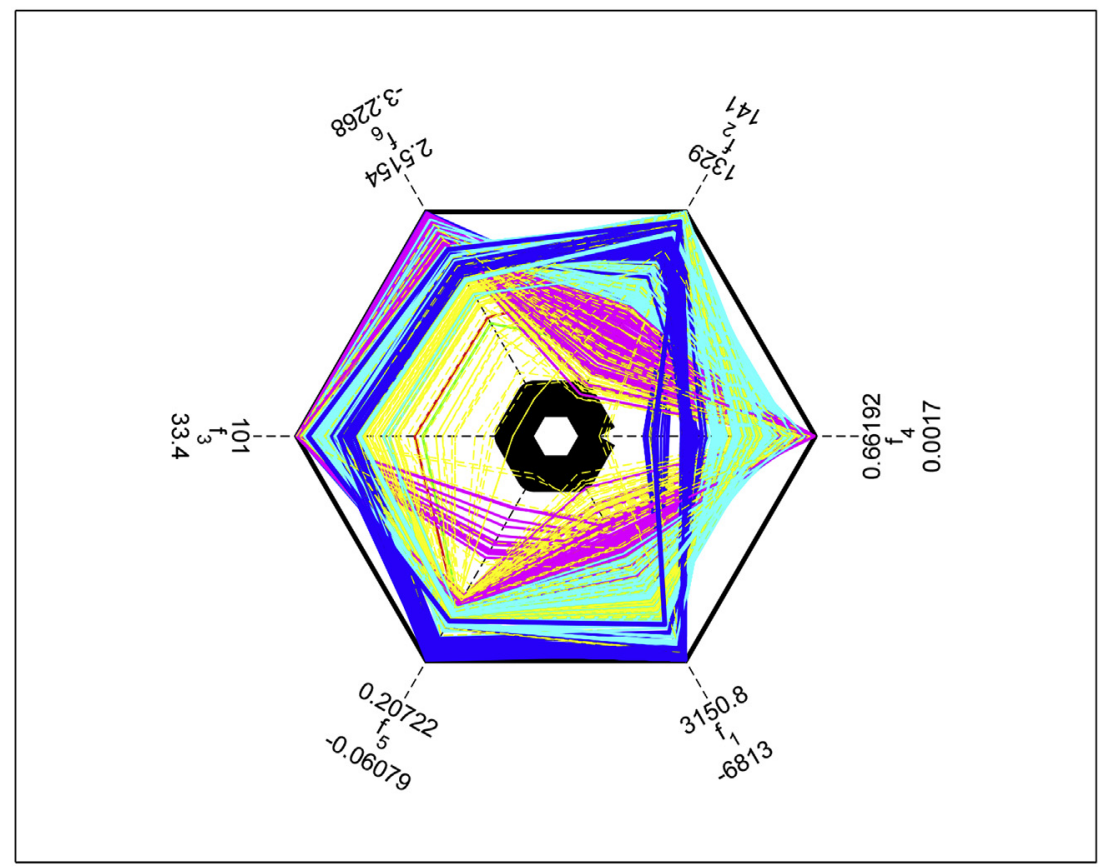

(b) Best case.

Fig. 8. Polar graph.

scenarios and for optimizing schedules volatility. The well-known economic indicator Sharpe Ratio was applied for evaluating a new cost-to-variability index.

It was verified a reasonable potential of improving the use of self-generation energy use and reducing systems peak load by using ESS based on PEVs located at SmartParks. Trade-offs between the use of PEVs batteries, which are an important environment issue, were discussed. Their use were mostly contrasted with the reduction of MG maximum peak load and its use was able also to minimize expected volatility on the power flow. It is expected that 


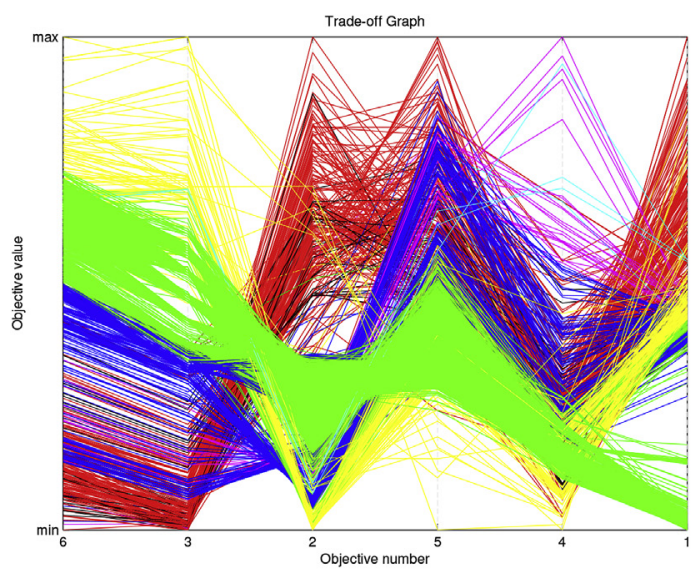

(a) Worst case.

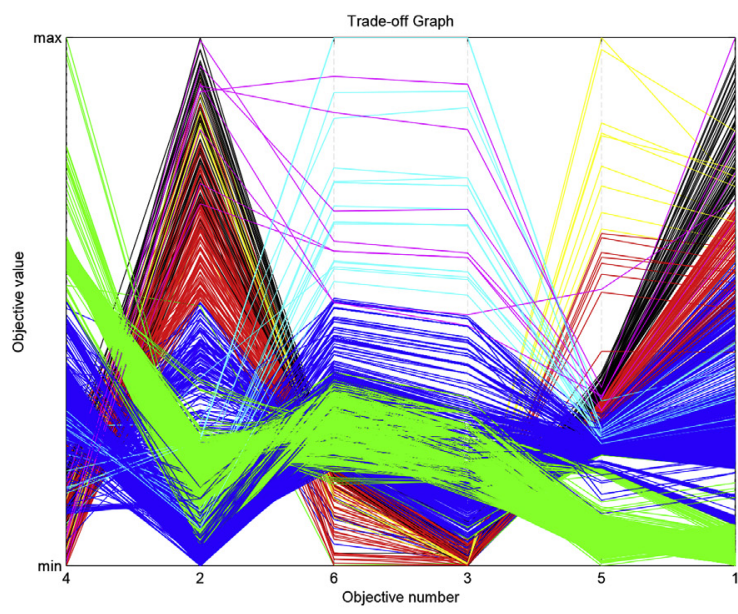

(b) Best case.

Fig. 9. Parallel coordinate plot.

the proposed model could be applied not only by MG users but also as a decision-making tool in order to assist smart-microgrid management.

\subsection{Extensions}

As future work the proposed model should be applied in other MG scenarios, including other renewable energy resources and larger scenarios. Uncertainties over PEVs availability could also be considered. The development of a metaheuristic based algorithm might provide an interest and flexible tool that can be applied over real large cases.

\section{Acknowledgment}

The authors would like to thank Brazilian agency CAPES, CNPq (grants 305506/2010-2, 552289/2011-6, 306694/2013-1 and 312276/2013-3), FAPEMIG (grant PPM CEX 497-13) and FP7 CORDIS, “New Horizons for Multi Criteria Decision Making”, for supporting the development of this work.

\section{References}

[1] C. Colson, M. Nehrir, A review of challenges to real-time power management of microgrids, in: Power Energy Society General Meeting, 2009. PES '09. IEEE, 2009, pp. 1-8, http://dx.doi.org/10.1109/PES.2009.5275343.

[2] X. Tan, Q. Li, H. Wang, Advances and trends of energy storage technology in microgrid, Int. J. Electr. Power Energy Syst. 44 (1) (2013) 179-191, http:// dx.doi.org/10.1016/j.ijepes.2012.07.015.

[3] P.F. Ribeiro, B.K. Johnson, M.L. Crow, A. Arsoy, Y. Liu, Energy storage systems for advanced power applications, Proc. IEEE 89 (12) (2001) 1744-1756.

[4] A.H. Fathima, K. Palanisamy, Optimization in microgrids with hybrid energy systems - a review, Renew. Sustain. Energy Rev. 45 (0) (2015) 431-446, http://dx.doi.org/10.1016/j.rser.2015.01.059.

[5] D. Olivares, A. Mehrizi-Sani, A. Etemadi, C. Canizares, R. Iravani, M. Kazerani, A. Hajimiragha, O. Gomis-Bellmunt, M. Saeedifard, R. Palma-Behnke, G. Jimenez-Estevez, N. Hatziargyriou, Trends in microgrid control, Smart Grid, IEEE Trans. 5 (4) (2014) 1905-1919, http://dx.doi.org/10.1109/ TSG.2013.2295514.

[6] Y. Levron, J. Guerrero, Y. Beck, Optimal power flow in microgrids with energy storage, Power Syst. IEEE Trans. 28 (3) (2013) 3226-3234.

[7] L.H. Macedo, J.F. Franco, M.J. Rider, R. Romero, Operação ótima de sistemas de armazenamento de energia em smart grids com fontes renováveis, in: Anais do XX Congresso Brasileiro de Automática, Belo Horizonte/MG, 2014.

[8] R. Rigo-Mariani, B. Sareni, X. Roboam, C. Turpin, Optimal power dispatching strategies in smart-microgrids with storage, Renew. Sustain. Energy Rev. 40 (0) (2014) 649-658. 10.1016/j.rser.2014.07.138.

[9] S. Mohammadi, S. Soleymani, B. Mozafari, Scenario-based stochastic operation management of microgrid including wind, photovoltaic, micro-turbine, fue cell and energy storage devices, Int. J. Electr. Power Energy Syst. 54 (0) (2014) 525-535, http://dx.doi.org/10.1016/j.ijepes.2013.08.004.

[10] P. Kou, D. Liang, L. Gao, J. Lou, Probabilistic electricity price forecasting with variational heteroscedastic gaussian process and active learning, Energy Convers. Manag. 89 (0) (2015) 298-308, http://dx.doi.org/10.1016/ j.enconman.2014.10.003.

[11] S. Wang, D. Yu, J. Yu, A coordinated dispatching strategy for wind power rapid ramp events in power systems with high wind power penetration, Int. J. Electr. Power Energy Syst. 64 (2015) 986-995, http://dx.doi.org/10.1016/ j.ijepes.2014.08.019.

[12] M. Delfanti, D. Falabretti, M. Merlo, Energy storage for $\{\mathrm{PV}\}$ power plant dispatching, Renew. Energy 80 (0) (2015) 61-72, http://dx.doi.org/10.1016/ j.renene.2015.01.047.

[13] J.P. Torreglosa, P. García, L.M. Fernández, F. Jurado, Energy dispatching based on predictive controller of an off-grid wind turbine/photovoltaic/hydrogen/ battery hybrid system, Renew. Energy 74 (2015) 326-336, http://dx.doi.org/ 10.1016/j.renene.2014.08.010.

[14] A. Tascikaraoglu, O. Erdinc, M. Uzunoglu, A. Karakas, An adaptive load dispatching and forecasting strategy for a virtual power plant including renewable energy conversion units, Appl. Energy 119 (2014) 445-453, http:// dx.doi.org/10.1016/j.apenergy.2014.01.020.

[15] J.P.T. ao, C.H. Antunes, A comparative analysis of meta-heuristic methods for power management of a dual energy storage system for electric vehicles, Energy Convers. Manag. 95 (2015) 281-296, http://dx.doi.org/10.1016/ j.enconman.2015.02.030.

[16] Z. Song, H. Hofmann, J. Li, J. Hou, X. Zhang, M. Ouyang, The optimization of a hybrid energy storage system at subzero temperatures: energy management strategy design and battery heating requirement analysis, Appl. Energy 159 (2015) 576-588, http://dx.doi.org/10.1016/j.apenergy.2015.08.120.

[17] H. Hemi, J. Ghouili, A. Cheriti, A real time fuzzy logic power management strategy for a fuel cell vehicle, Energy Convers. Manag. 80 (2014) 63-70, http://dx.doi.org/10.1016/j.enconman.2013.12.040.

[18] E. Alvarez, A. Lopez, J. Gómez-Aleixandre, N. de Abajo, On-line Minimization of Running Costs, Greenhouse Gas Emissions and the Impact of Distributed Generation Using Microgrids on the Electrical System, Sustainable Alternative Energy (SAE), 2009 IEEE PES/IAS Conference on, 2009, pp. 1-10, http:// dx.doi.org/10.1109/SAE.2009.5534847.

[19] C. Colson, M. Nehrir, C. Wang, Ant Colony Optimization for Microgrid Multiobjective Power Management, in: Power Systems Conference and Exposition, 2009, PSCE'09. IEEE/PES, 2009, pp. 1-7.

[20] H. Kanchev, D. Lu, B. Francois, V. Lazarov, Smart Monitoring of a Microgrid Including Gas Turbines and a Dispatched Pv-based Active Generator for Energy Management and Emissions Reduction, Innovative Smart Grid Technologies Conference Europe (ISGT Europe), 2010 IEEE PES, 2010, pp. 1-8, http:// dx.doi.org/10.1109/ISGTEUROPE.2010.5638875.

[21] J.P. Fossati, A. Galarza, A. Martín-Villate, L. Fontán, A method for optimal sizing energy storage systems for microgrids, Renew. Energy 77 (0) (2015) 539-549, http://dx.doi.org/10.1016/j.renene.2014.12.039.

[22] W.F. Sharpe, The sharpe ratio, J. Portfolio Manag. 21 (1) (1994) 49-58, http:// dx.doi.org/10.3905/jpm.1994.409501.

[23] V. Chow, C.W. Lai, Conditional sharpe ratios, Finance Res. Lett. 12 (0) (2015) 117-133, http://dx.doi.org/10.1016/j.frl.2014.11.001.

[24] S.C. Manchester, L.G. Swan, D. Groulx, Regenerative air energy storage for remote wind-diesel micro-grid communities, Appl. Energy 137 (0)) (2015) 490-500, http://dx.doi.org/10.1016/j.apenergy.2014.06.070.

[25] G. Comodi, A. Giantomassi, M. Severini, S. Squartini, F. Ferracuti, A. Fonti, 
742

V.N. Coelho et al. / Renewable Energy 89 (2016) 730-742

D.N. Cesarini, M. Morodo, F. Polonara, Multi-apartment residential microgrid with electrical and thermal storage devices: experimental analysis and simulation of energy management strategies, Apple. Energy 137 (0) (2015) 854-866, http://dx.doi.org/10.1016/j.apenergy.2014.07.068.

[26] B. Zakeri, S. Syri, Electrical energy storage systems: A comparative life cycle cost analysis, Renew. Sustain. Energy Rev. 42 (0) (2015) 569-596, http:// dx.doi.org/10.1016/j.rser.2014.10.011.

[27] P. Tinador, Superconducting Magnetic Energy Storage; Status and Perspective, IEEE/CSC\&ESAS European Superconductivity News Forum, No. 3, 2008.

[28] G. Venayagamoorthy, P. Chakravarty, Optimal Fuzzy Logic Based Coordination Controller for Improved Transient Stability of a Smart Grid, Fuzzy Systems (FUZZ-IEEE), 2014 IEEE International Conference on, 2014, pp. 346-353, http://dx.doi.org/10.1109/FUZZ-IEEE.2014.6891824.

[29] W. Kempton, J. Tomić, Vehicle-to-grid power fundamentals: calculating capacity and net revenue, J. Power Sources 144 (1) (2005) 268-279.

[30] Intelligent unit commitment with vehicle-to-grid - a cost-emission optimezation, J. Power Sources 195 (3) (2010) 898-911.

[31] R. Romeo, O. Micheloud, Power quality of actual grids with plugin electric vehicles in presence of renewable and micro-grids, Renew. Sustain. Energy Rev. 46 (0) (2015) 189-200, http://dx.doi.org/10.1016/j.rser.2015.02.014.

[32] P. Papadopoulos, S. Skarvelis-Kazakos, I. Grau, L. Cipcigan, N. Jenkins, Electric vehicles' impact on british distribution networks, Electro. Syst. Transp. IET 2 (3) (2012) 91-102, http://dx.doi.org/10.1049/iet-est.2011.0023.

[33] T. Hong, J. Wilson, J. Xe, Long term probabilistic load forecasting and normalization with hourly information, Smart Grid, IEEE Trans. 5 (1) (2014) 456-462.

[34] R. Weron, Electricity price forecasting: A review of the state-of-the-art with a look into the future, Int. J. Forecast. 30 (4) (2014) 1030-1081.

[35] Y. Chang, J. Wang, X. Wang, Review on probabilistic forecasting of wind power generation, Renew. Sustain. Energy Rev. 32 (0) (2014) 255-270, http:// dx.doi.org/10.1016/j.rser.2014.01.033.

[36] M. Zamo, O. Mestre, P. Arbogast, O. Pannekoucke, A benchmark of statistical regression methods for short-term forecasting of photovoltaic electricity production. part II: probabilistic forecast of daily production, Sol. Energy 105 (0) (2014) 804-816, http://dx.doi.org/10.1016/j.solener.2014.03.026.
[37] L.D. Monache, S. Alessandrini, Chapter 12-probabilistic Wind and Solar Power Predictions, in: L.E. Jones (Ed.), Renewable Energy Integration, Academic Press, Boston, 2014, pp. 149-158, http://dx.doi.org/10.1016/B978-0-12407910-6.00012-0.

[38] EirGrid National Control Center. [link]. URL http://www.eirgrid.com/ operations/systemperformancedata/systemdemand/.

[39] N. Lu, Q. Tang, J. Chang, W. Fan, J. Lu, A hybrid forecasting model with parameter optimization for short-term load forecasting of micro-grids, Apple. Energy 129 (0) (2014) 336-345.

[40] V. Coelho, F. Guimaraes, A. Reis, I. Coelho, B. Coelho, M. Souza, A Heuristic Fuzzy Algorithm Bio-inspired by Evolution Strategies for Energy Forecasting Problems, Fuzzy Systems (FUZZ-IEEE), 2014 IEEE International Conference on, 2014, pp. 338-345, http://dx.doi.org/10.1109/FUZZ-IEEE.2014.6891794.

[41] H.G. Beyer, H.P. Schwefel, Evolution strategies - a comprehensive introducton, Nat. Compute. 1 (2002) 3-52.

[42] O. Ledoit, W. M, Robust performance hypothesis testing with the sharpe ratio, J. Empire. Finance 15 (5) (2008) 850-859, http://dx.doi.org/10.1016/ j.jempfin.2008.03.002.

[43] A.H. Land, A.G. in, An automatic method of solving discrete programming problems, Econ. J. Econ. Soc. (1960) 497-520.

[44] T. Lust, J. Teghem, Two-phase pareto local search for the biobjective traveling salesman problem, J. Heuristics 16 (2010) 475-510.

[45] A.R. de Freitas, P.J. Fleming, F.G. Guimarães, Aggregation trees for visualization and dimension reduction in many-objective optimization, Inf. Sci. 298 (0) (2015) 288-314, http://dx.doi.org/10.1016/j.ins.2014.11.044.

[46] I.M. Coelho, P.L.A. Munhoz, M.N. Haddad, V.N. Coelho, M.M. Silva, M.J.F. Souza, L.S. Ochi, A Computational Framework for Combinatorial Optimization Problems, VII ALIO/EURO Workshop on Applied Combinatorial Optimization, Porto, 2011, pp. 51-54.

[47] V.N. Coelho, M.J.F. Souza, I.M. Coelho, F.G. Guimaraes, T. Lust, R.C. Cruz, Multiobjective approaches for the open-pit mining operational planning problem, Electron. Notes Discrete Math. 39 (0) (2012) 233-240.

[48] M.J.F. Souza, I.M. Coelho, S. Ribs, H.G. Santos, L.H.C. Merschmann, A hybrid heuristic algorithm for the open-pit-mining operational planning problem, Eur. J. Oper. Res. EJOR 207 (2010) 1041-1051. 